\title{
Vertical Structure of Selected Turbulence Characteristics above an Urban Canopy
}

\author{
C. Feigenwinter, R. Vogt, and E. Parlow
}

With 12 Figures

Received September 26, 1997

Revised February 15, 1998

\section{Summary}

In this paper the results of an urban measurement campaign are presented. The experiment took place from July 1995 to February 1996 in Basel, Switzerland. A total of more than 2000 undisturbed 30-minute runs of simultaneous measurements of the fluctuations of the wind vector $u^{\prime}, v^{\prime}, w^{\prime}$ and the sonic temperature $\theta_{s}^{\prime}$ at three different heights $(z=36,50$ and $76 \mathrm{~m}$ a.g.1.) are analysed with respect to the integral statistics and their spectral behaviour. Estimates of the zero plane displacement height $d$ calculated by the temperature variance method yield a value of $22 \mathrm{~m}$ for the two lower levels, which corresponds to $0.92 \mathrm{~h}$ (the mean height of the roughness elements). At all three measurement heights the dimensionless standard deviation $\sigma_{w} / u_{*}$ is systematically smaller than the Monin-Obukhov similarity function for the inertial sublayer, however, deviations are smaller compared to other urban turbulence studies. The $\sigma_{\theta} / \theta_{*}$ values follow the inertial sublayer prediction very close for the two lowest levels, while at the uppermost level significant deviations are observed. Profiles of normalized velocity and temperature variances show a clear dependence on stability. The profile of friction velocity $u_{*}$ is similar to the profiles reported in other urban studies with a maximum around $z / h=2.1$. Spectral characteristics of the wind components in general show a clear dependence on stability and dimensionless measurement height $z / h$ with a shift of the spectral peak to lower frequencies as thermal stability changes from stable to unstable conditions and as $z / h$ decreases. Velocity spectra follow the $-2 / 3$ slope in the inertial subrange region and the ratios of spectral energy densities $S_{w}(f) / S_{u}(f)$ approach the value of $4 / 3$ required for local isotropy in the inertial subrange. Velocity spectra and spectral peaks fit best to the well established surface

layer spectra from Kaimal et al. (1972) at the uppermost level at $z / h=3.2$.

\section{Introduction}

It is a well known problem, that methods for estimating the turbulent exchange, which were developed for rather smooth, homogeneous surfaces, actually can not be transferred to the urban boundary layer, where the surface is rough and inhomogeneous. In spite of that, due to a lack of better knowledge, the semi-empirical methods of the Monin-Obukhov similarity (MOS) theory are still used in flow and diffusion modelling (Beniston, 1987; Frenzen et al., 1987; Gross, 1989; Gryning and Lyck, 1984; Hanna and Chang, 1992), though its validity must be questioned.

The urban boundary layer can be divided into a roughness sublayer (RS) close to the roughness elements, followed by the inertial sublayer (IS) and topped by the (convective) mixed layer (ML) (Raupach et al., 1991). The RS and the IS together are also known as the surface layer. The RS extends up to 2-5 times the height of the roughness element $h$. The air flow in this layer is three dimensional due to the strong influences of the local building structures. Higher up in the IS the influences of a larger area are mixed in such a 
way, that the turbulent characteristics in the IS represent an area of average roughness. The well founded MOS relations can be applied to describe the turbulent fluxes and statistics in the IS, and therefore, the exchange of momentum, heat and mass can be calculated.

A first and often used approach to the problem is to investigate and to describe the deviations of the turbulent and spectral characteristics from their "ideal" values. Few studies deal with observations of the turbulence structure of the air flow over built-up areas. One of the assumptions made in MOS theory is, that the turbulent fluxes are constant with height. This is obviously not the case in the present study. So if i.e. the friction velocity $u_{*}$ is not constant with height, the Monin-Obukhov length $L$ also becomes essentially local and so does in consequence the stability parameter $z / L$. Similarly, the flux of sensible heat is found to be non-constant with height. Also the profiles of momentum flux and sensible heat flux observed at the present site depend strongly on stability. As a possible solution of the problem, the concept of local scaling is applied. Högström et al. (1982) and Rotach (1993b) found the concept of local scaling (local momentum flux values are used for normalization) to be valid for the description of velocity variances and wind-shear within the upper part of the urban roughness sublayer. However, the normalized standard deviations of vertical wind velocity are systematically smaller compared to MOS prediction as also is reported by Clarke et al. (1982), Rotach (1991) and Roth and Oke (1993b). Rotach (1993a) and Oikawa and Meng (1995) found similar profiles of $u_{*}$, which show a maximum at the supposed border between the RS and the IS. Despite the inhomogeneity and the roughness of an urban surface, Roth and Oke (1993a) reported spectral/ cospectral characteristics which differed only slightly to data from the homogeneous surface layer.

The aim of this campaign was to provide a reliable dataset to investigate the vertical structure of turbulence and organized motion above an urban canopy. The whole data set is analysed within the Monin-Obukhov similarity framework. Nondimensionalized and spectral properties of the turbulent flow are compared to standard surface layer relationships, and espe- cially the dependence of the observed relations of height and stability is investigated and discussed.

\section{Experimental Setup and Data Processing}

The data analysed in this paper were collected during a special measurement campaign as a part of the BASTA-project (BAsler STAdtklima) from July 1995 to February 1996 in the inner city of Basel, Switzerland. In order to extend the probing of the urban boundary layer above the roughness sublayer, turbulence instrumentation was mounted on a $50 \mathrm{~m}$ antenna tower, which has its basement at $24 \mathrm{~m}$ above street level. The tower has a triangle cross section with a sidelength of $1.25 \mathrm{~m}$ at the bottom and $0.5 \mathrm{~m}$ at the top. Within $500 \mathrm{~m}$ around the tower, the percentage of area covered by buildings over total area is $\approx 50 \%$ and the average building height is $\approx 24 \mathrm{~m}$.

Three ultrasonic anemometer-thermometers (sonics) measured at three heights the fluctuations of the wind vector $\left(u^{\prime}, v^{\prime}, w^{\prime}\right)$ and the sonic temperature $\left(\theta_{s}^{\prime}\right)$. At the two lower levels, the sonics were mounted at the end of a horizontal $6 \mathrm{~m}$ boom, at the uppermost level, the instrument was mounted right on the top. The data were sampled at $20.83 \mathrm{~Hz}$ and all raw data were stored for subsequent analysis. Additionally two profiles of wind speed $(u)$, and one profile of air temperature $(\theta)$ and humidity $(q)$ were measured, each profile consisting of six heights. Wind direction $(\alpha)$ was determined at three levels, and net radiation $\left(R_{n}\right)$ was measured at $64 \mathrm{~m}$. The profile data were stored as $10 \mathrm{~min}$ averages on a data logger (Campbell 21X). See Table 1 and Fig. 1 for an overview of the experimental setup.

The raw data of each sonic were sampled via serial port and stored as transit counts on three PCs, which have been synchronized for time at least once within three days. Maximum deviations were in the range of \pm 1.25 seconds per day. The raw data were scaled using a matrix calibration derived from wind tunnel experiments (Vogt, 1995). All three sonics were recalibrated after the measurement campaign in a wind tunnel in March 1996 and were also intercompared during a field campaign in April 1996 (Vogt et al., 1997). To minimize tower effects and to prevent for flow distortion by sonic geometry only data from an undistributed wind sector $\left(224^{\circ}<\alpha\right.$ 
Table 1. Instrumental Setup (see also Fig. 1)

\begin{tabular}{llll}
\hline Variable & Instrument & Manufacturer & Height [m] a.g.l. \\
\hline$u$ & cup anemometer & Vaisala WAA15 & $75,64,50,42,36,31$ \\
$u, \alpha$ & propeller vane & Young & $75,50,36$ \\
$\theta, q$ & thermistor, capacitive sensor & Vaisala HMP35A in ventilated radiation shield & $75,64,50,42,36,31$ \\
$R_{n}$ & pyrradiometer & Schenk Mod. 8111 & 64 \\
$u^{\prime}, v^{\prime}, w^{\prime}, \theta_{s}^{\prime}$ & ultrasonic anemometer thermometer & Gill-Solent, R2, asymmetric head & $76,50,36$ \\
\hline
\end{tabular}

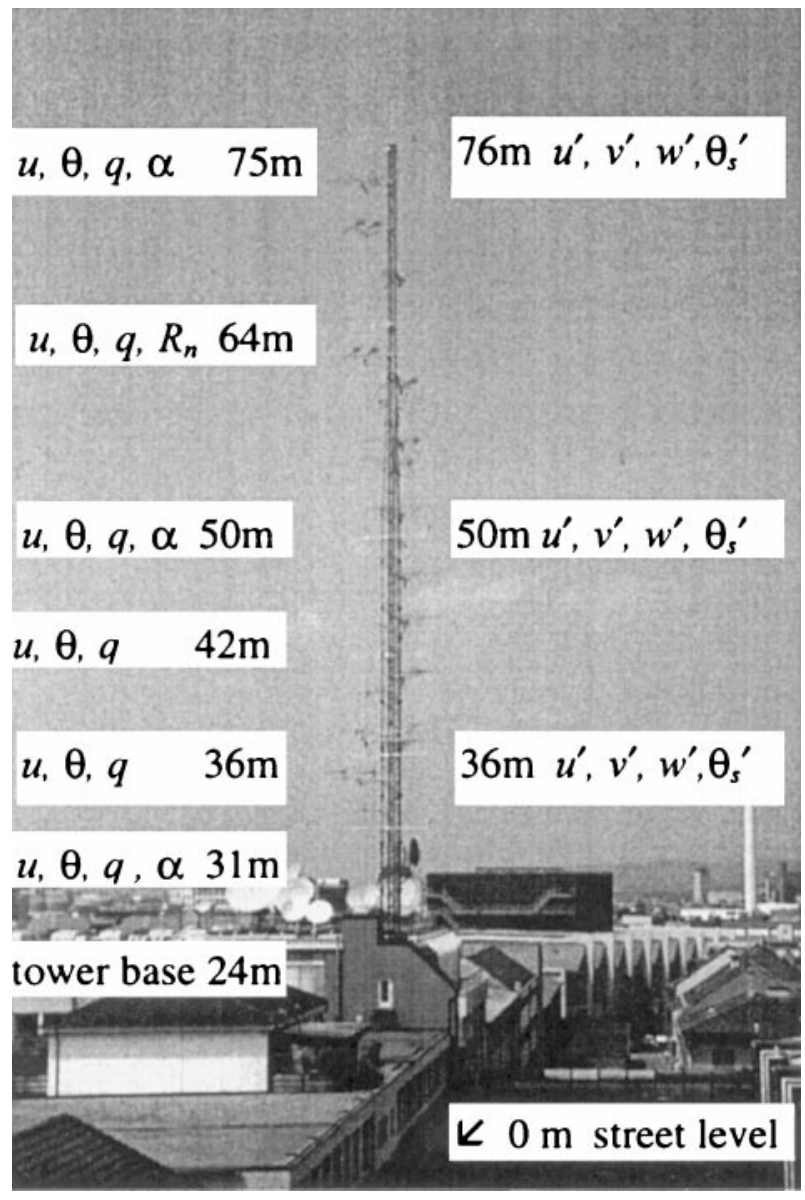

Fig. 1. The measurement tower in the inner city of Basel, Switzerland. For instruments setup refer to Table 1

$<103^{\circ}$ ) were selected for the analysis. Additionally, runs with mean wind speeds lower than $2 \mathrm{~ms}^{-1}$ at the uppermost measurement level were not included in the analysis. This resulted in a total of more than 1000 analysed 53-minute runs ( $2^{16}$ samples) for the spectral characteristics and more than 2000 30-minute runs for the analysis of the integral statistics and profiles (see also Table 4).

\section{Results and Discussion}

\subsection{Zero Plane Displacement Height}

The zero plane displacement height $d$ was determined by the temperature variance method described in Rotach (1994). Under certain conditions the surface of a city can be regarded as thermally homogeneous, even though it is dynamically rough. Therefore the measurement of the dimensionless temperature variance $\sigma_{\theta} / \theta_{*}$ can be used for the determination of $d$ providing that the same $d$ is valid for the flux of momentum as well as for the flux of latent heat. According to MOS theory the following function describes the behaviour of $\sigma_{\theta} / \theta_{*}$ for unstable conditions in the SL (from de Bruin et al., 1993; after Wyngaard et al., 1971; and Tillmann, 1972):

$\frac{\sigma_{\theta}}{\theta_{*}}=-C_{1}\left(1-C_{2} \frac{z-d}{L}\right)^{-\frac{1}{3}}$,

where $\theta_{*}$ denotes the temperature scale $\left(\theta_{*}=-\overline{w^{\prime} \theta^{\prime}} / u_{*}\right), L$ the Monin-Obukhov length, and the constants $C_{1}$ and $C_{2}$ are 2.9 and 28.4.

To get an estimate of $d$, the difference between measured and calculated values is minimized according to Eq. (2) by varying $d$.

$e r r=\left(\frac{1}{N} \sum_{i=1}^{N}\left(\frac{\sigma_{\theta_{i}}}{\theta_{*_{i}}}-\left(\frac{\sigma_{\theta}}{\theta_{*}}\right)_{i(1)}\right)^{2}\right)^{\frac{1}{2}}$

In Eq. (2), $N$ is the number of measurements and err is the value to be minimized.

Table 2 gives an overview on the results of the temperature variance method for the undisturbed sector of wind direction. The resulting averaged $d$ estimates for the lower two measurement heights are $21 \mathrm{~m}$ and $23.5 \mathrm{~m}$ correspond quite well whereas at the topmost level we find an unrealistic high value of about $40 \mathrm{~m}$. The reason 
Table 2. Values for the Zero Plane Displacement Height d Calculated by the Temperature Variance Method for the Selected Wind Direction Sectors (number of runs per sector in brackets)

\begin{tabular}{|c|c|c|c|c|c|c|c|c|c|}
\hline Height & $240^{\circ} \pm 15^{\circ}$ & $270^{\circ} \pm 15^{\circ}$ & $300^{\circ} \pm 15^{\circ}$ & $\begin{array}{l}\text { Azimuth } \\
330^{\circ} \pm 15^{\circ}\end{array}$ & $0^{\circ} \pm 15^{\circ}$ & $30^{\circ} \pm 15^{\circ}$ & $60^{\circ} \pm 15^{\circ}$ & $90^{\circ} \pm 15^{\circ}$ & $\begin{array}{l}\text { Average } \\
225^{\circ}-105^{\circ}\end{array}$ \\
\hline$z / \mathrm{h}=3.2$ & $47.0(12)$ & $42.0(68)$ & $39.0(26)$ & $39.0(99)$ & $37.0(50)$ & $35.0(2)$ & 34.0 (4) & $48.0(51)$ & 40.1 \\
\hline$z / h=2.1$ & $29.5(15)$ & 23.5 & $26.5(39)$ & $22.5(106)$ & $19.5(47)$ & - & $19.5(5)$ & $23.5(53)$ & 23.5 \\
\hline$z / h=1.5$ & $25.5(18)$ & $22.0(120)$ & $22.0(47)$ & 20.0 & 19.0 & $18.5(3)$ & $24.5(3)$ & $16.5(56)$ & 21.0 \\
\hline
\end{tabular}

for this could be the different source area of this sensor that probably does not fullfill the conditions for thermal homogeneity. A zero plane displacement of $22 \mathrm{~m}$ corresponds to 0.92 times the building height $h$, which seems comparatively high to values for vegetation canopies, which are in the range of $2 / 3$ to $3 / 4 h$. However, considering that urban buildings stand very close together and are stiff compared to a vegetation canopy, so that the wind profile can not penetrate very deep into the urban canopy, and also taking into account the excellent agreement of the results from the lower two sonics, it is justified to use a $d$-value of $22 \mathrm{~m}$ for all further calculations.

\subsection{Integral Statistics and Profiles}

\subsubsection{Profiles of Velocity and Temperature Standard Deviations and Friction Velocity}

In Figs. 2, 3 and 4 average profiles of velocity and temperature standard deviations are shown for the four stability classes stable $(z / L>0.05)$, neutral $(-0.05 \leq z / L \leq 0.05)$, slightly unstable $(-0.5 \leq z / L \leq-0.05)$ and unstable $(z / L \leq$ $-0.05)$. Standard deviations $\sigma_{u}, \sigma_{v}$ and $\sigma_{w}$ and the friction velocity $u_{*}$ are scaled with the $u_{*}-$ value from the uppermost measurement level at $z / h=3.2$ and error bars indicate the run-to-run variability. In the near neutral case profiles of $\sigma_{u}$ and $\sigma_{v}$ in Fig. $2 \mathrm{a}$ and $2 \mathrm{~b}$ are nearly uniform, whereas $\sigma_{w}$ increases slightly with height. Taking into account the small scatter, the values correspond surprisingly well to the recommended values of $2.4,1.9$ and 1.25 by Panofsky and Dutton (1984) for $\sigma_{u} / u_{*}, \sigma_{v} / u_{*}$ and $\sigma_{w} / u_{*}$, respectively, for neutral conditions and flat terrain. Under unstable conditions the profiles of $\sigma_{u}$ and $\sigma_{v}$ decrease with height, whereas for stable conditions $\sigma_{u}$ increases and $\sigma_{v}$ has a relative

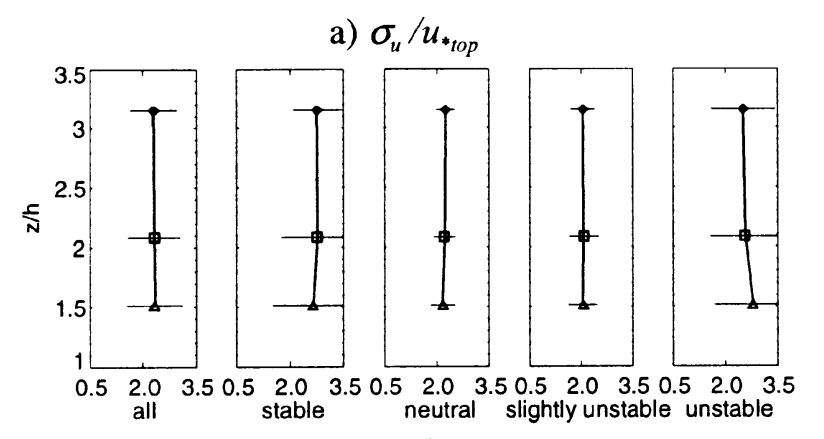

b) $\sigma_{v} / u_{\text {top }}$

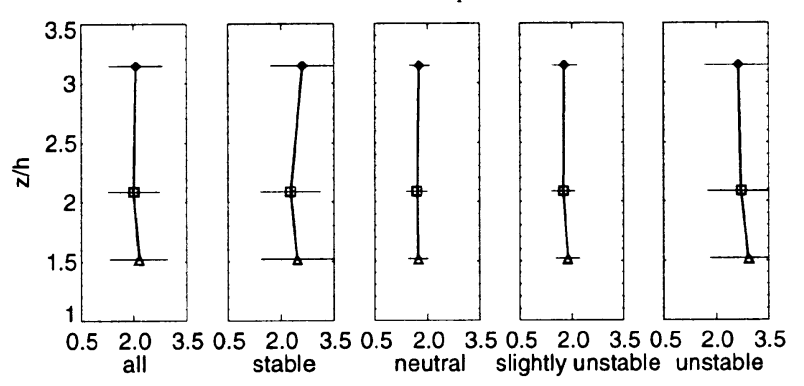

c) $\sigma_{w} / u_{* \text { top }}$

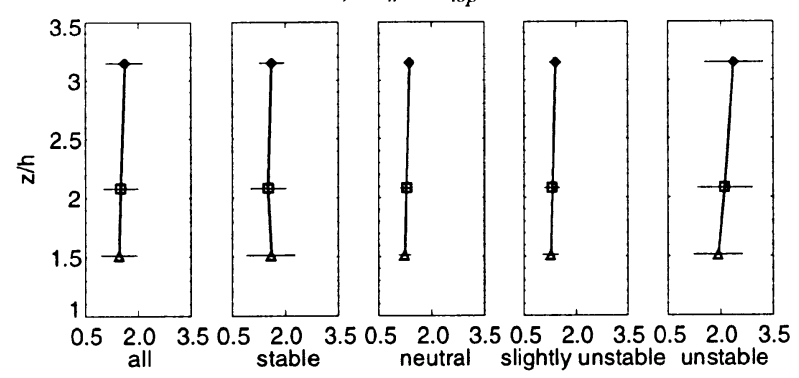

Fig. 2. Scaled profiles of standard deviations of wind components a) $\sigma_{u} / u *_{\text {top }}$ b) $\sigma_{v} / u *_{\text {top }}$ and c) $\sigma_{w} / u *_{\text {top }}$

minimum at $z / h=2.1$. The scaled vertical velocity variance $\sigma_{w}$ shows an increase with height from neutral to unstable conditions, differences become larger as instability increases. This is in contrast to the observation of Rotach (1995), who reported a decrease of $\sigma_{w}$ with increasing height for near-neutral stratifications. 


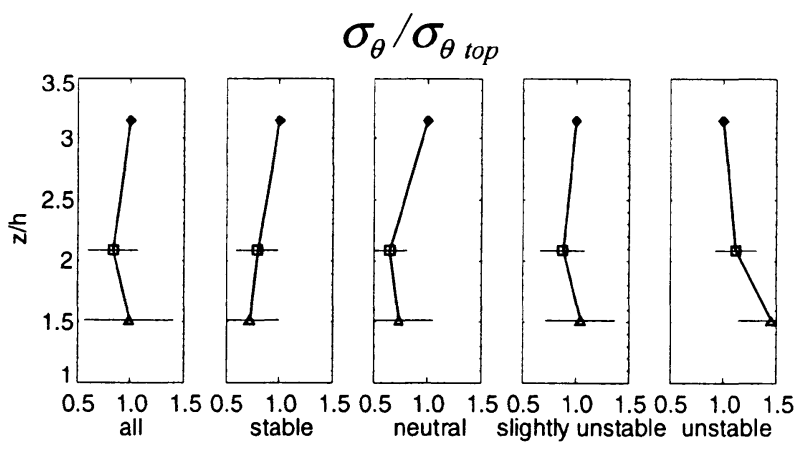

Fig. 3. Scaled profiles of standard deviations of temperature

The profile of temperature variance normalized by the $\sigma_{\theta}$ value of the uppermost level (Fig. 3) shows a strong decrease of $\sigma_{\theta}$ with increasing height for unstable conditions. At $z / h=1.5$ fluctuations are about $50 \%$ higher than at $z / h=$ 3.2. For stable and neutral stratifications, $\sigma_{\theta}$ is reduced in the lower levels compared to the top level. This increase of $\sigma_{\theta}$ between $z / h=2.1$ and $z / h=3.2$ for slightly unstable to stable stratifications supports the idea of different thermal source areas, as we would expect $\sigma_{\theta}$ to decrease with height.

Profiles of friction velocity $u_{*}$ are displayed in Fig. 4. For unstable stratification $u_{*}$ decreases slightly between the two upper levels, whereas for the remaining cases, a significant increase with height between the two lower levels can be observed. Under stable stratification the maximum occurs at $z / h=2.1$ at the second measurement level. Considering these profiles and taking into account Rotach's (1993a) suggestion to consider the RS as the layer within $u_{*}$ increases from zero to the IS value, we can set the upper boundary of the RS somewhere around the second measurement level at $z / h=2.1$. A similar

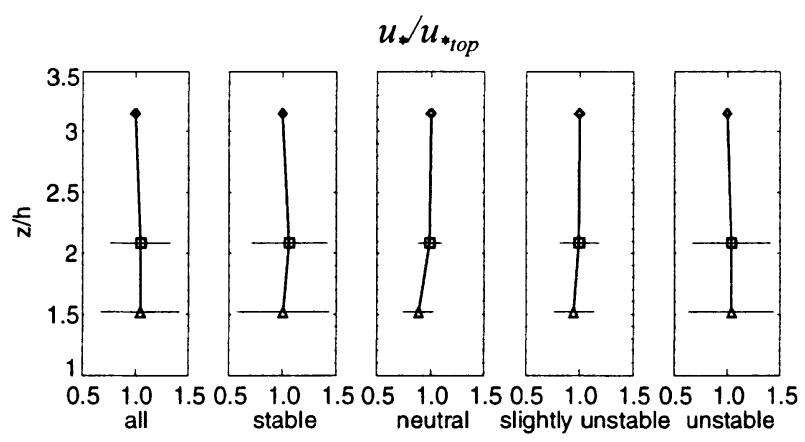

Fig. 4. Scaled profiles of friction velocity $u *$ profile of $u_{*}$ is reported in Oikawa and Meng (1995).

\subsubsection{Normalized Standard Deviations}

The analysis shows that the dimensionless standard deviation for the vertical wind velocity $\sigma_{w} / u_{*}$ is systematically smaller for all three measurement heights than the values predicted by the MOS function for $\sigma_{w} / u_{*}$ for "ideal" surfaces:

$\frac{\sigma_{w}}{u_{*}}=C_{1}\left(1-C_{2} \frac{z-d}{L}\right)^{\frac{1}{3}}$.

The coefficients $C_{1}$ and $C_{2}$ in Eq. (3) are taken from Panofsky and Dutton (1984) as 1.25 and 3. A similar behaviour is reported by Clarke et al. (1982), Roth and Oke (1993b), Rotach (1993b) and Oikawa and Meng (1995), however, deviations to the reference in our study are smaller, which is shown in Fig. 5 left. Note that local values of $u_{*}$ and $L$ are taken. As listed in Table 3, all urban studies report smaller values for $C_{1}$ and $C_{2}$ in Eq. (3), therefore, one can conclude that over rough urban surfaces an enhanced mechanical production of vertical velocity fluctuations is established.

Since the zero plane displacement height $d$ was determined by the temperature variance method (see previous section), the $\sigma_{\theta} / \theta_{*}$ values are practically forced to follow the MOS function in Eq. (1). This works quite well for the two lower levels, however, the large anomalies at the uppermost level at $z / h=3.2$ in Fig. 5 right suggests, that thermal homogeneity is not given for the source area of this sensor. This is also in agreement to the statement derived from the $\sigma_{\theta}$-profile in Fig. 3 in the previous section and the failure of the temperature variance method for this measurement level.

\subsection{Spectral Characteristics}

Time series of 53 minutes ( $2^{16}$ samples) have been chosen for spectral analysis. Before calculating the spectra, data were corrected for possible non-stationarities by linear detrending and tapered with a cosine taper prior to the discrete Fourier transformation (Stull, 1988; Kaimal and Finnigan, 1994). 

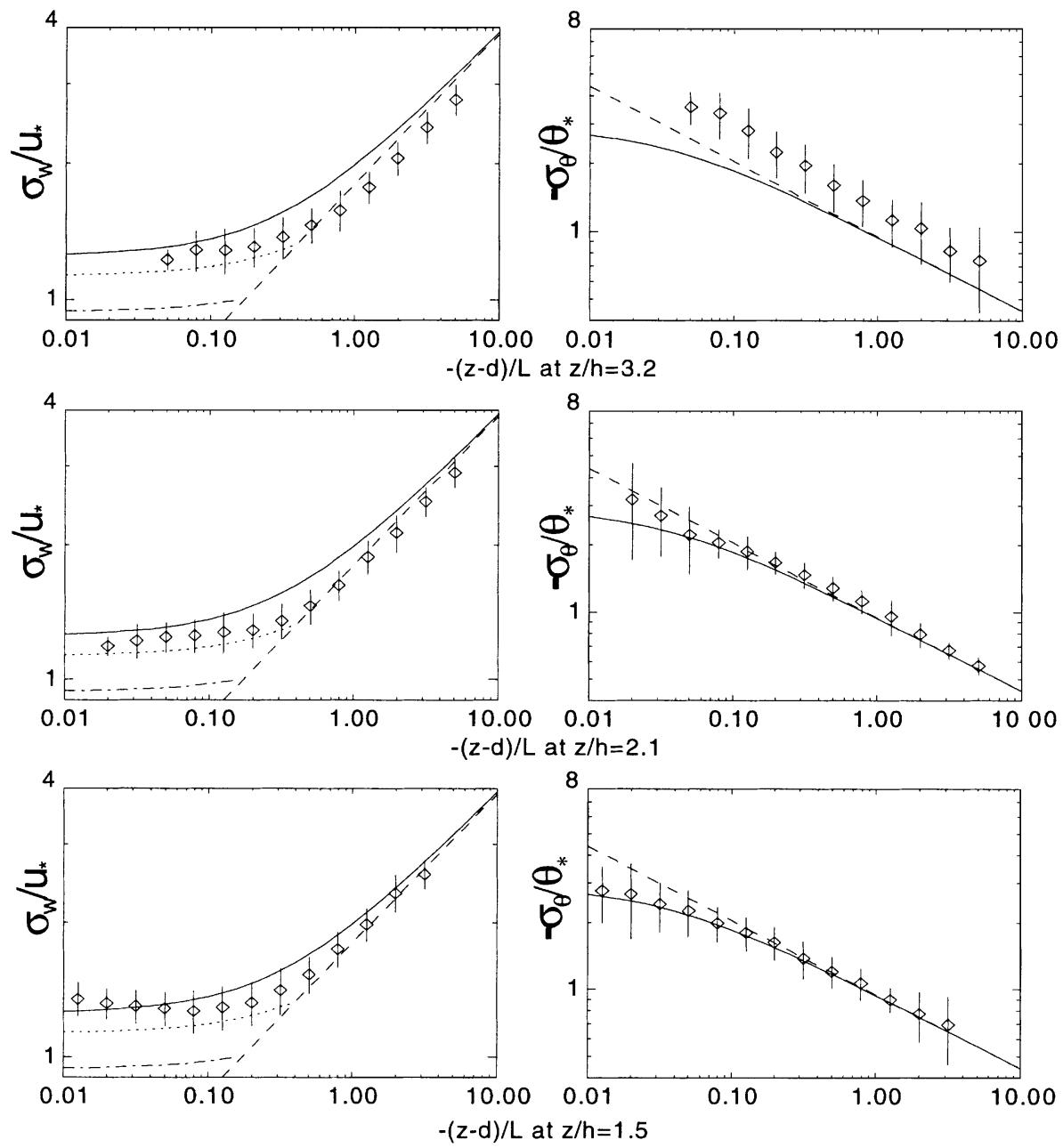

Fig. 5. Left column: $\sigma_{w} / u_{*}$ values for unstable conditions. Plotted are class averages with one standard deviation as error bar. Functional relationships are from Panofsky and Dutton (1984) (solid), Clarke et al. (1982) (dotted) and Rotach (1993b) (dashed-dotted). Right column: same as left, but for $\sigma_{\theta} / \theta_{*}$. Functional relationship is after de Bruin et al. (1993) (solid). The free convection limit is indicated by the dashed line

Table 3. Comparison of Values $C_{1}$ and $C_{2}$ in Eq. (3) from Different Studies

\begin{tabular}{lllll}
\hline$C_{1}$ & $C_{2}$ & $z / h$ & surface & study \\
\hline 1.25 & 3 & & "ideal" & Panofsky and Dutton (1984) \\
1.13 & 1.56 & $z / h \approx 4$ & average value of four urban sites, $0 \geq z / L \geq-5$ & Clarke et al. (1982) \\
0.94 & 1.29 & $1.27 \leq z / h \leq 1.55$ & urban site, $0 \geq z / L \geq-1$ & Rotach (1993b) \\
1.16 & 2.36 & $z / h=3.2$ & urban site, $-0.02 \geq z / L \geq-8$ & this study \\
1.14 & 2.82 & $z / h=2.1$ & & \\
1.18 & 2.95 & $z / h=1.5$ & & \\
\hline
\end{tabular}

The presented spectra and cospectra are normalized by their respective variances and covariances and plotted against the non-dimensional frequency $f=n(z-d) / \bar{u}$, where $n$ denotes the natural frequency in $\mathrm{Hz}$ and $\bar{u}$ the mean wind speed over the sampling time. The averaged mean wind speed over all runs was $3.9 \mathrm{~m} / \mathrm{s}$ at the uppermost level. Note that all spectra and frequencies are normalized by their local values. All single spectra were fitted by a cubic spline and then averaged into the four stability classes. The number of runs per class are listed in Table 4. Spectral and cospectral peak frequencies are compared in Table 5 and Table 6 to other urban turbulence studies.

Additionally the well established Kansas spectra from Kaimal et al. (1972) for neutral conditions are plotted in the following figures for 
Table 4. Stability Classes and Number of 53-minute Runs for Composite Spectra Calculation

\begin{tabular}{lllll}
\hline & Stable & Neutral & Weakly unstable & Unstable \\
$z / h$ & $z / L>0.05$ & $-0.05 \leq z / L \leq 0.05$ & $-0.5 \leq z / L<-0.05$ & $z / L<-0.5$ \\
\hline 1.5 & 63 & 386 & 709 & 147 \\
2.1 & 79 & 244 & 702 & 280 \\
3.2 & 173 & 164 & 511 & 455 \\
\hline
\end{tabular}

Table 5. Spectral Peak Frequencies of Wind Components $u, v$ and $w$ above Urban Surfaces (Except Kaimal et al., 1972) from Various Studies

\begin{tabular}{|c|c|c|c|c|c|}
\hline \multirow[t]{2}{*}{ Study } & \multicolumn{3}{|c|}{ Peak frequency } & \multirow{2}{*}{$\begin{array}{l}\text { Height } \\
z / h\end{array}$} & \multirow[t]{2}{*}{ Stability } \\
\hline & $u$ & $v$ & $w$ & & \\
\hline Kaimal et al. (1972) & 0.045 & 0.142 & 0.45 & & neutral \\
\hline \multirow[t]{2}{*}{ Rotach (1995) } & $0.1 . .1 .2$ & & $\approx 0.65$ & 1.55 & $0>z / L>-1$ \\
\hline & $0.05 . .0 .5$ & & $\approx 1.4$ & 1.27 & $0>z / L>-1$ \\
\hline \multirow[t]{2}{*}{ Högström et al. (1982) } & $5.053 \pm 0.017$ & $0.24 \pm 0.037$ & $0.54 \pm 0.12$ & 3.5 & $-0.2<z / L<0.2$ \\
\hline & $0.101 \pm 0.039$ & $0.11 \pm 0.02$ & $0.35 \pm 0.08$ & 1.3 & $-0.2<z / L<0.2$ \\
\hline Oikawa and Meng (1995) & $0.02 . .0 .05$ & & $0.1 . .0 .4$ & 2.5 & $-0.08>z / L>-0.23$ \\
\hline Roth and Oke (1993a) & $\approx 0.02$ & $\approx 0.14$ & $\approx 0.15$ & 2.65 & $-0.05>z / L>-1.8$ \\
\hline \multirow[t]{3}{*}{ This study } & $\approx 0.044$ & $\approx 0.131$ & $\approx 0.38$ & 3.2 & $-0.05>z / L>-0.5$ \\
\hline & $\approx 0.029$ & $\approx 0.085$ & $\approx 0.31$ & 2.1 & $-0.05>z / L>-0.5$ \\
\hline & $\approx 0.015$ & $\approx 0.044$ & $\approx 0.25$ & 1.5 & $-0.05>z / L>-0.5$ \\
\hline
\end{tabular}

Table 6. (Co)Spectral Peak Frequencies of Temperature $\theta$, Momentum Flux $\overline{u^{\prime} w^{\prime}}$ and Kinematic Heat Flux $\overline{w^{\prime} \theta^{\prime}}$ above Urban Surfaces (except Kaimal et al., 1972) from Various Studies

\begin{tabular}{|c|c|c|c|c|c|}
\hline \multirow[t]{2}{*}{ Study } & \multicolumn{3}{|c|}{ Peak frequency } & \multirow{2}{*}{$\begin{array}{l}\text { Height } \\
z / h\end{array}$} & \multirow[t]{2}{*}{ Stability } \\
\hline & $\theta$ & $u^{\prime} w^{\prime}$ & $w^{\prime} \theta^{\prime}$ & & \\
\hline Kaimal et al. (1972) & 0.063 & 0.07 & 0.09 & & neutral \\
\hline Rotach $(1991,1995)$ & distorted & $\approx 0.25$ & $\approx 0.25$ & 1.55 & $-0>z / L>-1$ \\
\hline Roth and Oke (1993a) & $\approx 0.04$ & $\approx 0.035$ & $\approx 0.045$ & 2.65 & $-0.05>z / L-1.8$ \\
\hline \multirow[t]{3}{*}{ This study } & distorted & $\approx 0.085$ & $\approx 0.16$ & 3.2 & $-0.05>z / L-0.5$ \\
\hline & $\approx 0.085$ & $\approx 0.044$ & $\approx 0.085$ & 2.1 & $-0.05>z / L>-0.5$ \\
\hline & $\approx 0.068$ & $\approx 0.055$ & $\approx 0.085$ & 1.5 & $-0.05>z / L>-0.5$ \\
\hline
\end{tabular}

comparison reasons. Note that the velocity spectra are scaled with the variance whereas the Kansas spectra scale with $u_{*}$, this explains the differences in the power level. The slopes of $-2 / 3$ and $-4 / 3$ in the following figures indicate the proportionality of the spectral and cospectral energy densities, respectively, to the inertial subrange laws predicted by Kolmogorov's hypothesis.

\subsubsection{Velocity Spectra}

In Figs. 6, 7 and 8 the composite spectra of the wind components $u, v$ and $w$ are shown for all 


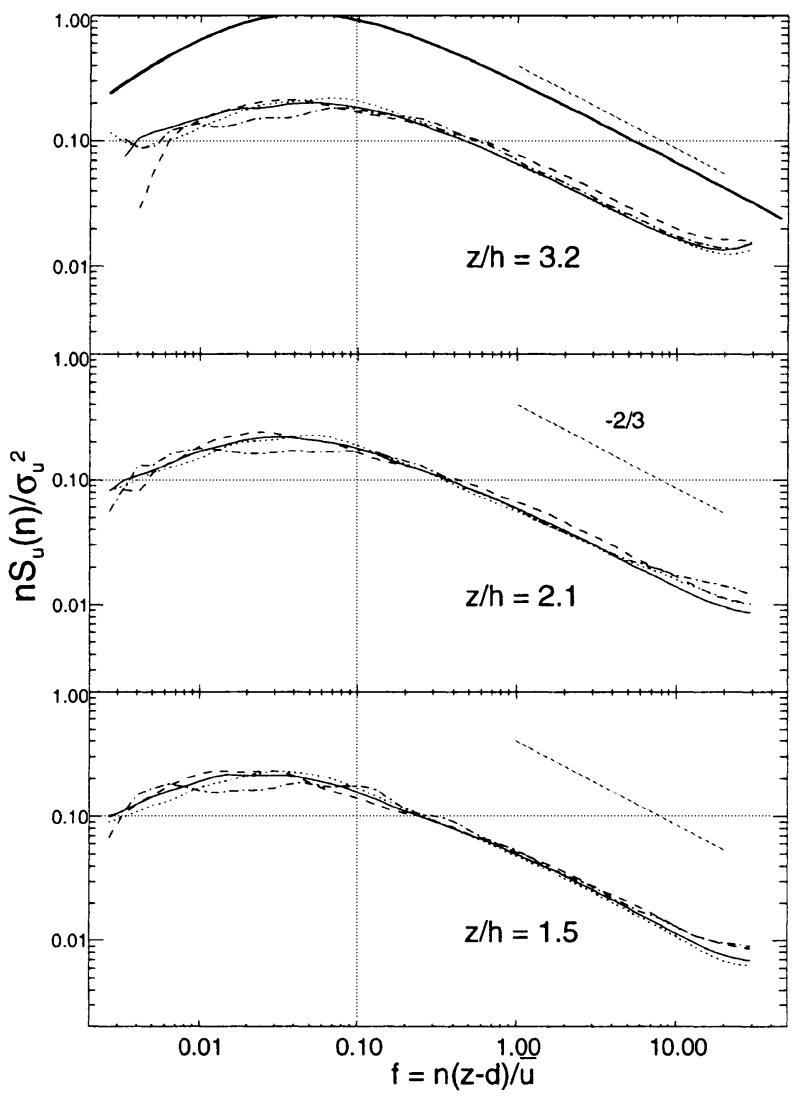

Fig. 6. Spectra of longitudinal wind component $u$ for stable (dashed-dotted), neutral (dotted), weakly unstable (solid) and unstable (dashed) conditions. Reference spectrum from Kaimal et al. (1972) (solid thick)

three measurement heights and the four stability classes. In the inertial subrange they all follow the $-2 / 3$ slope. In contrast to the lateral and vertical velocity spectra, the spectra of the longitudinal velocity $u$ in Fig. 6 do not exhibit a strong stability dependence. Only a weak tendency for a peak shift to lower frequencies can be observed at each height as stratification becomes more unstable. Spectral peaks for weakly unstable conditions occur in then region of $f_{m} \approx 0.044$ for $z / h=3.2$ and shift to lower frequencies for all stability classes as we come closer to the roughness elements. Since $f_{m}=$ $(z-d) / \lambda_{m}$ by virtue of Taylor's hypothesis, the dominant eddy scale or peak wavelength $\lambda_{m}$ for the longitudinal component $u$ ranges from $1200 \mathrm{~m}$ at $z / h=3.2$ to $969 \mathrm{~m}$ at $z / h=2.1$ and $960 \mathrm{~m}$ at $z / h=1.5$ for weakly unstable conditions.

The spectra of the lateral and vertical wind components $v$ and $w$ in Figs. 7 and 8 show a clear

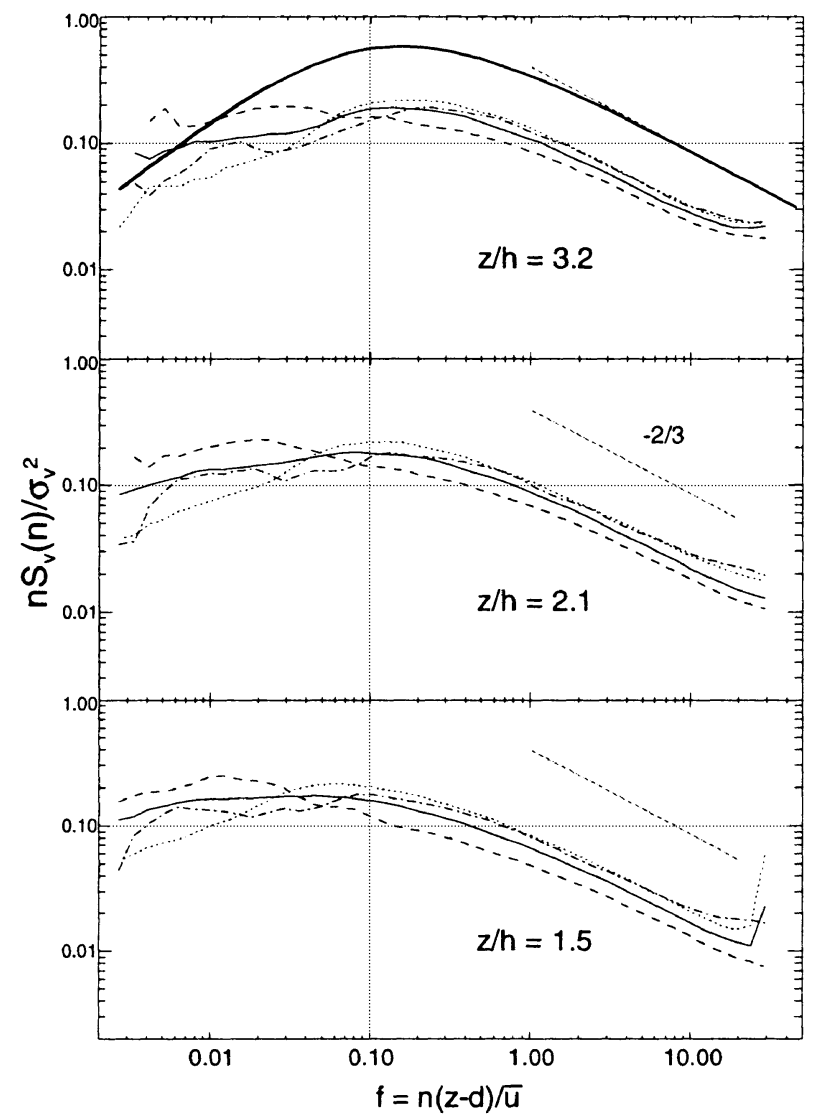

Fig. 7. As Fig. 6 but for lateral wind component $v$

stability dependence at all three measurement heights. Spectral energy densities for unstable conditions are significantly higher in the low frequency range and the spectral peaks are shifted to lower frequencies as instability increases. In the inertial subrange, the $-2 / 3$ slope is well established, but in contrast to the longitudinal wind component spectral densities in the inertial subrange are lowest for unstable conditions and they increase as stratification becomes more and more stable. As observed with the $u$-spectra, the peak frequencies of the $v$ - and $w$-spectra shift to lower values as $z / h$ decreases. Peak frequencies $f_{m}$ of $w$-spectra in the weakly unstable case are $0.38,0.31$ and 0.25 from top to bottom. This corresponds to dominant eddy scales $\lambda_{m}$ of $141 \mathrm{~m}, 91 \mathrm{~m}$ and $58 \mathrm{~m}$.

Since the peak wavelengths $\lambda_{m}$ decrease for all three wind components as we approach the surface from the uppermost measurement level, the dimensions of the dominant eddy structures 


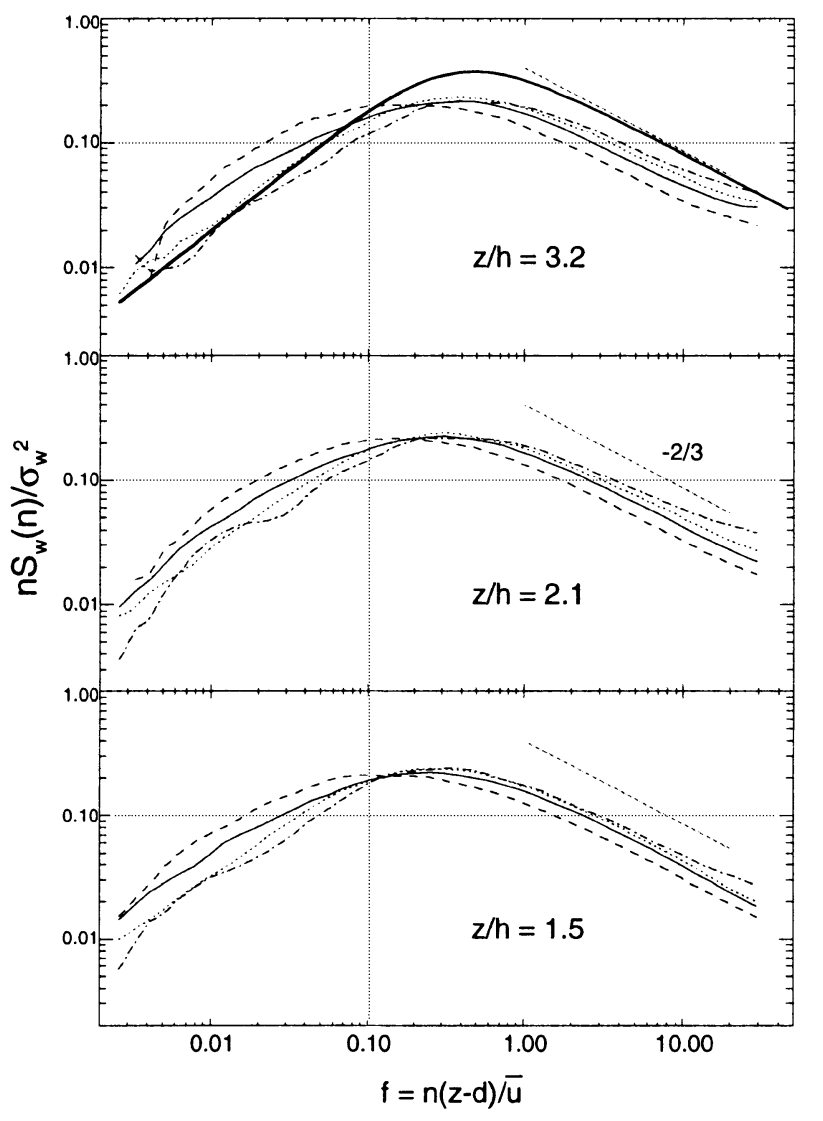

Fig. 8. As Fig. 6 but for vertical wind component $w$

become smaller. The peak frequencies of the surface layer reference spectra from Kaimal et al. (1972) fit best to the uppermost level at $z / h=3.2$ for all three wind components, which suggests that this measurement level is above the roughness sublayer within the inertial sublayer. In Table 5 the observed spectral peak spectral peak frequencies of wind components are compared to values reported in other urban studies. The scatter between the values for the same variable in quite large, but one has to consider the different measurement heights and the different "urban" surfaces. Roth and Oke (1993a) and Oikawa and Meng (1995) measured over relatively uniform suburban areas (mean building height $h=8.5 \mathrm{~m}$ and $7 \mathrm{~m}$, respectively) with a percentage of area covered by buildings of about 25\%, while Högström et al. (1982) and Rotach (1995) were investigating european cities ( $h=15 \mathrm{~m}$ and $20 \mathrm{~m}$, respectively) with about $50 \%$ of total area covered by buildings, which is

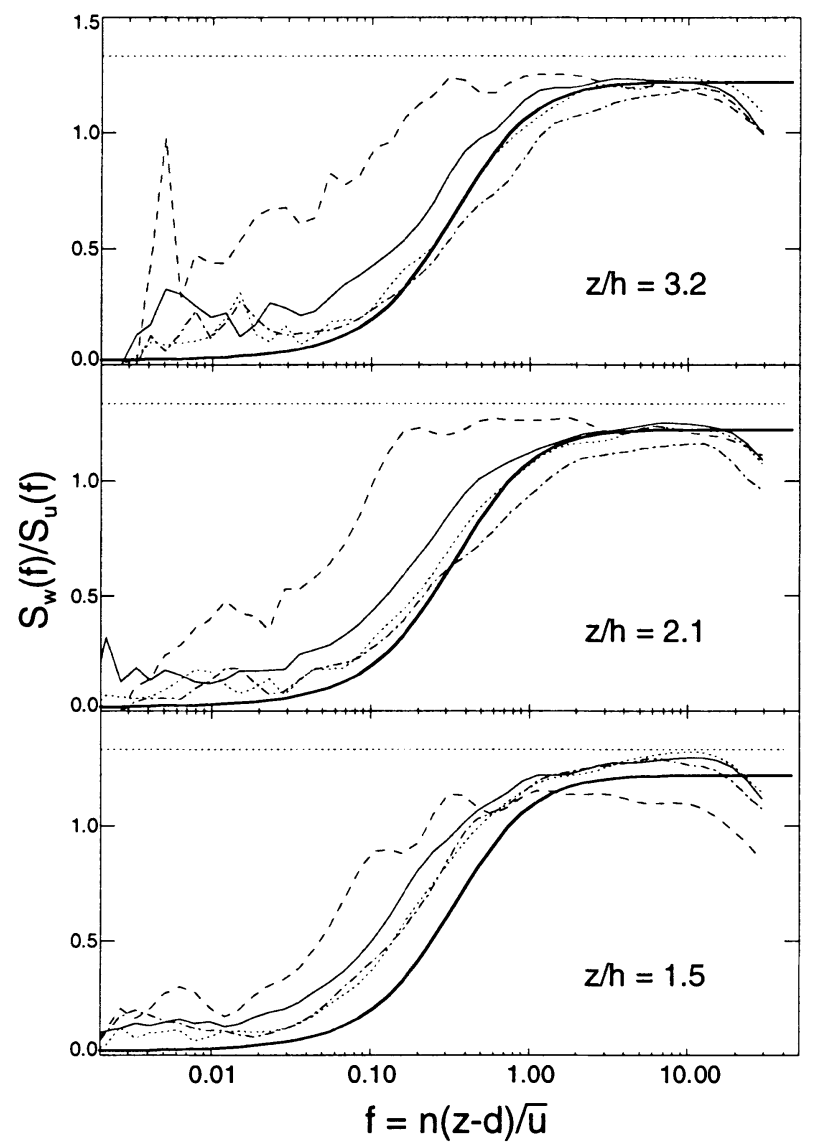

Fig. 9. As Fig. 6 but for ratios of spectral energy densities $S_{w}(f) /(S)_{u}(f)$. Reference curve solid thick from Kaimal et al. (1972)

similar to the present study. Additionally the number of runs analysed in the study is significantly larger in all studies mentioned above (see Table 4).

Horizontal and vertical velocity composite spectra exhibit the predicted $-2 / 3$ slope at the high frequency range and therefore, one could conclude, that an inertial subrange is observed within the urban roughness sublayer. As an additional test based on Kolmogorov's hypothesis concerning local isotropy in this frequency range, the ratio of the vertical to the longitudinal spectral energy densities $S_{w}(f) / S_{u}(f)$ is expected to approach 4/3. This ratio is drawn in Fig. 9 for all measurement heights and stability classes. Note that the Kansas spectra are not adjusted to provide the 4/3 ratio (Kaimal and Finnigan, 1994). This is in good agreement with the $z / h=3.2$ level, however the $z / h=1.5$ curve approaches the $4 / 3$ line best. Rotach (1995) reported values for $S_{w}(f) / S_{u}(f)$ smaller than 1 
at $z / h=1.55$ and concluded that there is no inertial subrange within the urban roughness sublayer. However the present data and the values from Högström et al. (1982), who reported $S_{w}(f) / S_{u}(f)$ ratios of 1.3 and 1.06 for $z / h=3.5$ and $z / h=1.3$, respectively, suggest that an inertial subrange is established.

\subsubsection{Temperature Spectra}

Temperature spectra in Fig. 10 are generally illdefined and flat for stable and neutral conditions at all heights and also for weakly unstable conditions at $z / h=3.2$. The increase of the spectral densities at the low frequency end of these spectra indicate a significant mesoscale component, which is also observed by Clarke et al. (1982). Rotach (1995) explains the higher spectral densities at low frequencies with warm bubbles rising sporadically from the street canyon. The fact that the increase of spectral densities at the low frequency end is most pronounced at the uppermost level supports the

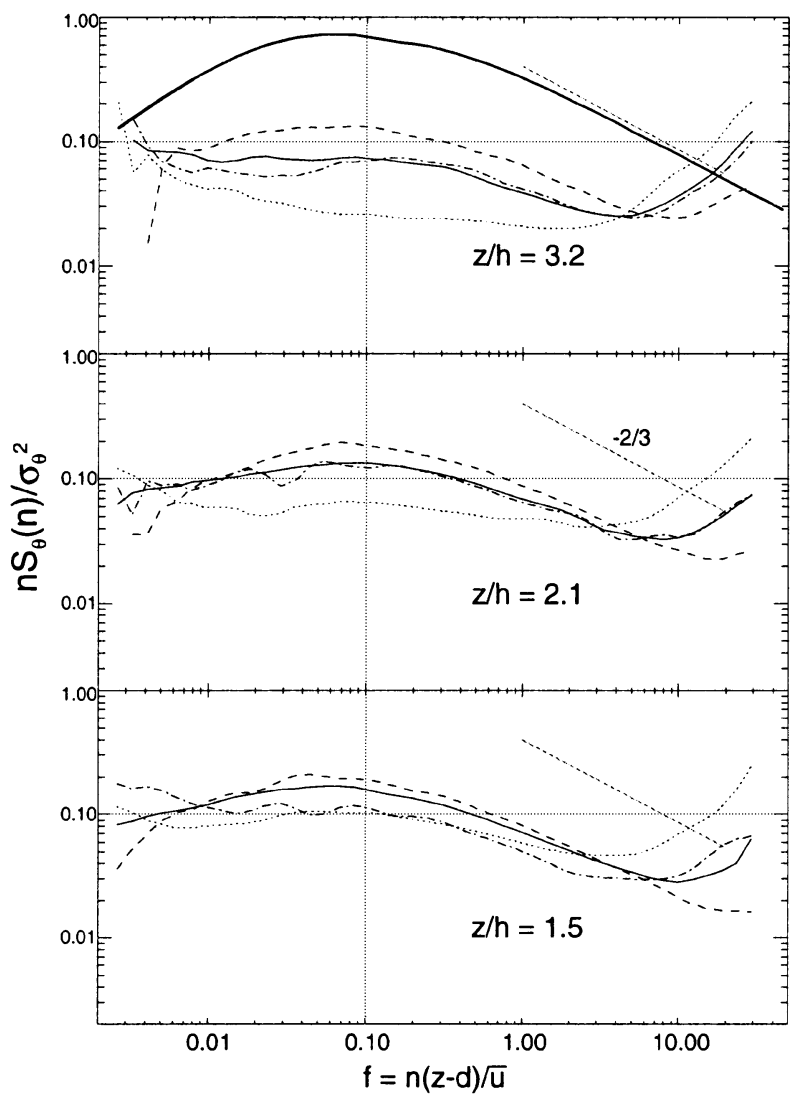

Fig. 10. As Fig. 6 but for sonic temperature $\theta_{s}$ idea of a different thermal regime at the uppermost measurement level as supposed in a previous section, though the interpretation of temperature spectra must be made with caution. A striking feature of all temperature spectra is their increase at the high frequency end at all heights and a stabilities at $f>5$, which might reflect a noisy signal. The $-2 / 3$ slope in the inertial subrange is only followed for unstable conditions, the corresponding peak frequencies are $0.085,0.069$ and 0.044 for $z / h=3.2,2.1$ and 1.5 , respectively.

\subsubsection{Cospectra}

As for temperature spectra there are not many studies that deal with cospectra over urban surfaces. Some of the few are compared to this study in Table 5. Because of the larger scatter and occasional negative values, the curve for stable conditions are not well formed, nevertheless they are plotted in Fig. 11, too. Cospectra of $u^{\prime} w^{\prime}$ are well established for neutral and

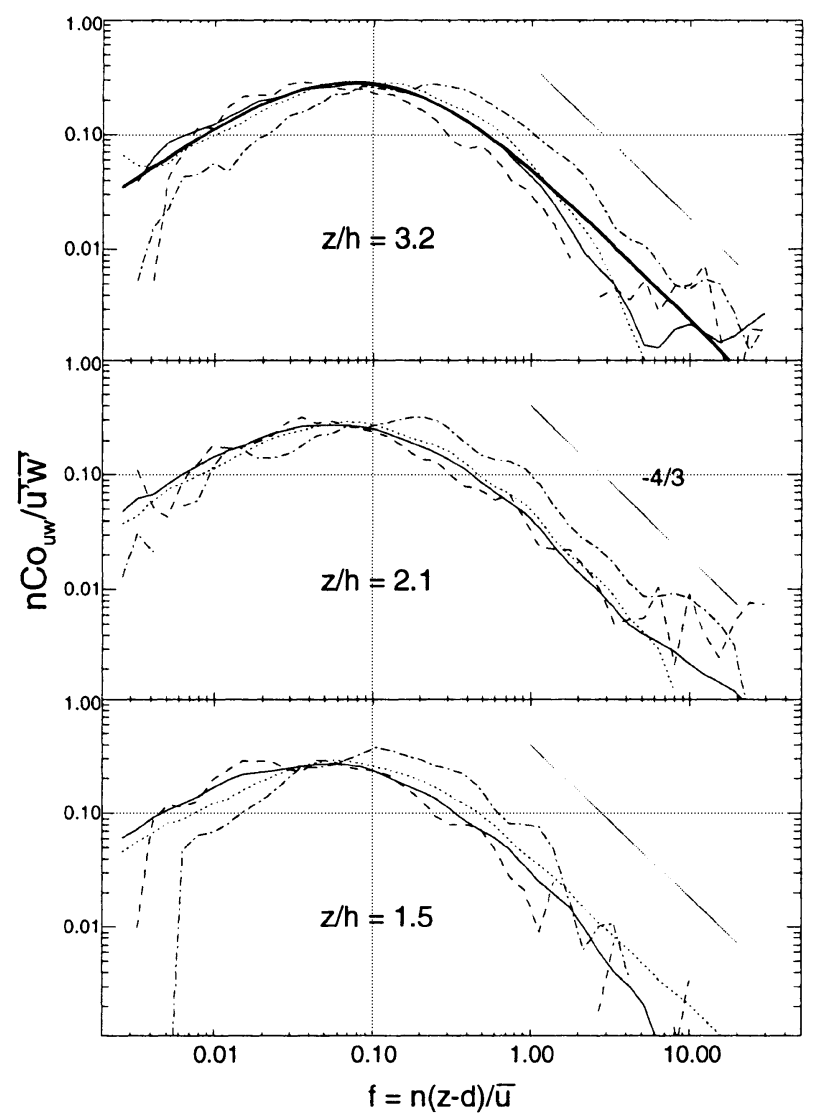

Fig. 11. As Fig. 6 but for Cospectra of $u^{\prime} w^{\prime}$ 


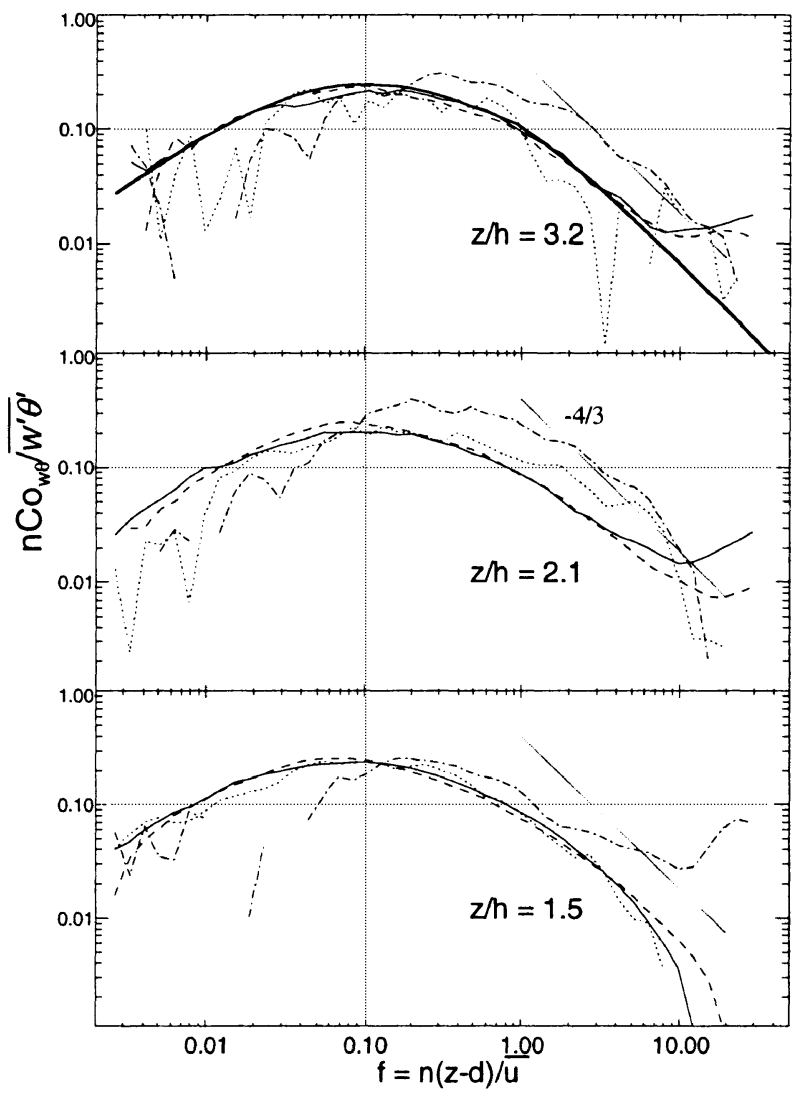

Fig. 12. As Fig. 6 but for Cospectra of $w^{\prime} \theta^{\prime}$

weakly unstable conditions, however the $-4 / 3$ slope is not given for the uppermost level in the inertial subrange and peak locations for the two lower levels are slightly shifted to lower values (Table 6). Cospectra of $w^{\prime} \theta^{\prime}$ in Fig. 12 behave very similar to those of $u^{\prime} w^{\prime}$, again the curves are not well defined at all measurement levels for the stable stability class and at $z / h=3.2$ and $z / h=$ 2.1 for the neutral class. Nevertheless the reference cospectrum agrees almost perfectly in shape and peak frequency with the weakly unstable cospectra of the two lower levels, whereas the peak frequency for $z / h=3.2$ is shifted to a higher value of $f_{m} \approx 0.16$. Compared to the velocity spectra, cospectra do not show that distinct height and stability dependence, what makes a clear interpretation difficult.

\section{Summary and Conclusions}

Simultaneously measured turbulent time series at three different heights $(z / h=1.5,2.1$ and 3.2$)$ above an urban canopy in Basel, Switzerland, were analysed within the Monin-Obukhov similarity framework and with respect to their spectral characteristics. Results are compared to previous studies made over urban surfaces.

1) Both $\sigma_{w} / u_{*}$ and $\sigma_{\theta} / \theta_{*}$ follow the predicted (-)1/3 power law dependence for free-convection. The normalized standard deviation $\sigma_{w} / u_{*}$ for unstable conditions is found to be systematically smaller at all three measurement heights than rural reference data, but larger than the values from comparable studies over urban surfaces (Clarke et al., 1982; Rotach, 1993b).

2) Scaled average profiles of standard deviations of the horizontal velocity components $\sigma_{u}$ and $\sigma_{v}$, and of temperature $\sigma_{\theta}$ show a clear stability dependence such as a decrease with height under unstable conditions and an increase with height under stable conditions. The standard deviation of the vertical component $\sigma_{w}$ is found to generally increase with height and differences become larger as instability increases.

3 ) The profiles of friction velocity $u_{*}$ are found to decrease continuously with height only under unstable conditions, whereas under weakly unstable to stable conditions $u_{*}$ shows a maximum at $z / h=2.1$. This seems to be characteristic for the urban roughness sublayer, as similar profiles are reported by Rotach (1993a) and Oikawa and Meng (1995).

4) Spectral peaks of wind component $u, v$ and $w$ show a clear height dependence such as a shift to higher frequencies as height increases. The predicted $-2 / 3$ slope in the inertial subrange region is generally well established and the ratio $S_{w} / S_{u}$ approaches a value of 1.22 at $z / h=2.1$. and 1.3 at $z / h=1.5$. This leads to the conclusion, that an inertial subrange is established though we are in the urban roughness sublayer. Peak frequencies fit best to the Kansas reference spectra at the uppermost measurement level at $z / h=3.2$, but are slightly lower. The spectra of $v$ and $w$ show an obvious shift to lower frequencies as instability increases, whereas the $u$-spectra show only a weak stability dependence.

5) Temperature spectra and cospectra of $w^{\prime} \theta^{\prime}$ are generally not well defined for stable and neutral stratifications. At the uppermost measurement level at $z / h=3.2$ temperature spectra are very flat also for weakly unstable and unstable conditions and do not show a distinct peak. 
6) Three facts suggests that the uppermost level at $z / h=3.2$ is probably ruled by another turbulent thermal regime than the two lower levels, namely: a) the failure of the temperature variance method for the determination of the zero plane displacement $d$, b) the significant deviation from MOS prediction for $\sigma_{\theta} / \theta_{*}$, and c) the flat and ill-defined temperature spectra.

Mechanical properties in the urban roughness sublayer such as profiles of velocity variances, non-dimensionalized velocity variances and spectra of wind componnents behave similar to rural reference data and other urban studies. They ar parametrized quite well within the MOS framework, if local scaling is applied. Parametrization of thermal properties in the urban roughness sublayer and inertial sublayer within the MOS framework however is not satisfactory, since different measurement levels show different behaviour to standard equations probably due to thermal inhomogeneity and/or different source areas.

Since this is first study that describes the vertical dependence of velocity spectra in the urban surface layer by the analysis of a large number of runs, there is certainly a need for more experiments in this field to confirm the results of this and previous studies. Thereby it should also to be taken in account that the structure of urban surfaces depends heavily on regional aspects, e.g. they are not of the same kind in Europe and in North America.

\section{Acknowledgements}

Funding for this study is provided by the Swiss National Science Foundation grants 20-40621.94 and 20-49490.96. Our thanks to Balcab Kabelfernsehen Basel AG, Basel, for giving us the permission to use the antenna tower and their facilities for our measurements, to M. W. Rotach from the Swiss Federal Institute of Technology, Dept. of Geography, Zürich, for his advice for the experimental setup and his institute for providing us with several instruments for this campaign.

\section{References}

Beniston, M., 1987: A numerical study of atmospheric pollution over complex terrain in Switzerland. Bound.Layer Meteor., 41, 75-96.

Clarke, J. F., Ching, J. K. S., Godowich, J. M., 1982: A study of turbulence in a urban environment. EPA 600-53-82062, EPA.
De Bruin, H. A. R., Koshiek, W., van den Hurk, B. J. J. M., 1993: A verification of some methods to determine the fluxes of momentum, sensible heat and water vapour using standard deviation and structure parameter of scalar meteorological quantities. Bound.-Layer Meteor., 63, 231-257.

Frenzen, G., Heimann, D., Wamser, M., 1987: Dokumentation des Regionalen Klimamodells (RKM) auf der Basis von FITNAH. DFVLR-Mitt. 87-07.

Gross, G., 1989: Numerical simulation of the nocturnal flow systems in the Freiburg area for different topographies. Beiträge zur Physik der Atmosphäre, 62, 57-72.

Gryning, S.-E., Lyck, E., 1984: Atmospheric dispersion from elevated sources in an urban area: comparison between tracer experiments and model calculations. J. Climate Appl. Meteor., 23, 651-660.

Hanna, S. R., Chang, J. C., 1992: Boundary-Layer parametrizations for applied dispersion modeling over urban areas. Bound.-Layer Meteor., 58, 229-259.

Högström, U., Bergström, H., Alexandersson, H., 1982: Turbulence characteristics in a near neutrally stratified urban atmosphere. Bound.-Layer Meteor., 23, 449-472.

Kaimal, J. C., Wyngaard, J. C., Izumi, J., Coté, O. R., 1972: Spectral characteristics of surface-layer turbulence. Quart. J. Roy. Meteor. Soc., 98, 563-589.

Kaimal, J. C., Finnigan, J. J., 1994: Atmospheric Boundary Layer Flows: Their Structure and Measurement. New York: Oxford University Press, 289 pp.

Oikawa, S., Meng, Y., 1995: Turbulence characteristics and organized motions in a suburban roughness sublayer. Bound.-Layer Meteor., 74, 289-312.

Panofsky, H. A., Dutton, J. A., 1984: Atmospheric Turbulence. Models and Methods for Engineering Applications. New York: John Wiley \& Sons, 397 pp.

Rotach, M. W., 1991: Turbulence within and above an Urban Canopy. ETH Diss. 9439, published as ZGS, Heft 45, Zürich: Verlag GGIETH, 240 pp.

Rotach, M. W., 1993a: Turbulence close to a rough urban surface. Part I: Reynolds stress. Bound.-Layer Meteor., 65, $1-28$.

Rotach, M. W., 1993b: Turbulence close to a rough urban surface. Part II: Variances and gradients. Bound.-Layer Meteor., 66, 75-92.

Rotach, M. W., 1994: Determination of the zero plane displacement in an urban environment. Bound.-Layer Meteor., 67, 187-193.

Rotach, M. W., 1995: Profiles of turbulence statistics in and above an urban street canyon. Atmos. Environ., 29, 1473-1486.

Roth, M., Oke, T. R., 1993a: Tubulent transfer relationships over an urban surface: I: Spectral characteristics. Quart. J. Roy. Meteor. Soc., 119, 1071-1104.

Roth, M., Oke, T. R., 1993b: Tubulent transfer relationships over an urban surface: II: Integral statistics. Quart. J. Roy. Meteor. Soc., 119, 1105-1120.

Stull, R. B., 1988: An Introduction to Boundary Layer Meteorology. Dordrecht: Kluwer Academic Publishers, $666 \mathrm{pp}$.

Tillmann, J. E., 1972: The indirect determination of stability, heat and momentum fluxes in the atmospheric boundary 
layer from simple scalar variables during dry unstable conditions. J. Appl. Meteor., 11, 783-792.

Vogt, R., 1995: Theorie, Technik and Analyse der experimentellen Flussbestimmung am Beispiel des Hartheimer Kiefernwaldes. Ein Beitrag zu den Energiebilanzuntersuchungen im REKLIP, stratus 3, Diss. Phil-. Nat. -Fak. Univ. Basel, Basel, 101 pp.

Vogt, R., Feigenwinter, C., Paw U. K. T., Pitacco, A., 1997: Intercomparison of ultrasonic anemometers, Preprints of the $12^{\text {th }}$ Symposium of Boundary Layers and Turbulence, Vancouver, Canada. Am. Met. Soc., 354-355.
Wang, J., 1992: Turbulence characteristics in an urban atmosphere of complex terrain. Atmos. Environ., 25A, 2717-2724.

Wyngaard, J. C., Coté, O. R., Izumi, Y., 1971: Local free convection, similarity, and the budgets of shear stress and heat flux. J. Atmos. Sci., 28, 1171-1182.

Authors' addresses: Dipl. Geogr. Christian Feigenwinter, Dr. Roland Vogt, and Prof. Dr. Eberhard Parlow, MCR Lab, Department of Geography, University of Basel, Spalenring 145, CH-4055 Basel, Switzerland. 